\title{
A Hybrid Numerical Scheme for Fractional-Order Systems
}

\author{
L. L. Ferrás $s^{1,2}$, N. J. Ford ${ }^{2}$, M. L. Morgado ${ }^{3(\bowtie)}$, and M. Rebelo ${ }^{4}$ \\ 1 Institute for Polymers and Composites/I3N \& CMAT-UM, \\ University of Minho, Guimarães, Portugal \\ 2 Department of Mathematics, University of Chester, Chester, UK \\ 3 CEMAT, Instituto Superior Técnico, \\ Universidade de Lisboa and Department of Mathematics, \\ University of Trás-os-Montes e Alto Douro, UTAD, Vila Real, Portugal \\ luisam@utad.pt \\ 4 Centro de Matemática e Aplicações (CMA) and Departamento \\ de Matemática, Faculdade de Ciências e Tecnologia, \\ Universidade NOVA de Lisboa, Caparica, Portugal
}

\begin{abstract}
In this work we present a hybrid numerical scheme for the solution of systems of fractional differential equations arising in several fields of engineering. The numerical scheme can deal with both smooth and non-smooth solutions, and, the idea behind the hybrid method is that of approximating the solution as a linear combination of nonpolynomial functions in a region near the singularity, and by polynomials in the remaining domain. The numerical method is then used to study fractional RC electrical circuits.
\end{abstract}

Keywords: Fractional differential equations $\cdot$ Caputo derivative Non-polynomial collocation method $\cdot$ Polynomial collocation method Electrical circuits

\section{Introduction}

Fractional differential equations are becoming a hot topic in mathematics and engineering, and, in the last few decades we have witnessed a mass generalization of classical models to their fractional version. The reason is that several physical systems rely on memory $[1,2]$ for their evolution, or, the fact that their rates of evolution need not to be classical derivatives of orders $1,2,3 \ldots$, but instead, can be something in between. Therefore, fractional models allow a better modeling of physical phenomena by capturing information that is lost when we go from smaller scales to the usual continuum approach.

For example, fractional calculus plays an important role in control systems based on proportional-integral-derivative controllers, known as PID, and commonly used in industry, in instruments and laboratory equipment [1]. In these processes we arrive at systems of fractional differential equations that may need to be solved numerically. 
Other important examples of application of fractional calculus are electric circuits. As shown in the work of Gómez-Aguilar et al. [3] the use of fractional derivatives allows a much better fit to experimental data (see that of spectroscopy applied to an RC circuit in [3]). Since the analytical solution of fractional differential equations is only available for a small number of cases, and due to the fact that when using discrete techniques it takes a large amount of time to transform data from one domain to the other, in their work [3], Gómez-Aguilar et al. used the Numerical Laplace Transform to convert the time domain into the frequency domain (and vice-versa).

Another possibility is to discretize the integral representation of the problem directly. Therefore, in this work, we are concerned with the numerical solution of the linear systems of ordinary differential equations:

$$
\begin{aligned}
& D^{\alpha} \mathbf{y}(t)=A \mathbf{y}(t)+\mathbf{F}(t), \quad t \in(0, T] \\
& \mathbf{y}(0)=\mathbf{y}_{\mathbf{0}},
\end{aligned}
$$

where $A$ is a constant matrix $A=\left[a_{i j}\right]_{i, j=1, \ldots, n}, \mathbf{y}=\left[\begin{array}{ll}y_{1} & y_{2} \ldots y_{n}\end{array}\right]^{T}, \mathbf{F}(t)=$ $\left[f_{1}(t) f_{2}(t) \ldots f_{n}(t)\right]^{T}$ and $\mathbf{y}_{\mathbf{0}}=\left[\begin{array}{ll}y_{01} & y_{02} \ldots y_{0 n}\end{array}\right]^{T}$, where $y_{0 i}=y_{i}(0), i=1, \ldots, n$. The order of the fractional derivative satisfies $0<\alpha<1$, and the fractional derivative is given in the Caputo sense, that is [2]:

$$
\frac{d^{\alpha} u(t)}{d t^{\alpha}}=\frac{1}{\Gamma(1-\alpha)} \int_{0}^{t}(t-s)^{-\alpha} u^{\prime}(s) d s .
$$

In this work we present a hybrid numerical scheme for the solution of systems of fractional differential equations (FDE) arising in different fields of engineering. The numerical scheme can deal with both smooth and non-smooth solutions, and, the idea behind the hybrid method is that of approximating the solution as a linear combination of non-polynomial functions in a region near the singularity [4], and by polynomials in the remaining domain.

The work is organized as follows. In Sect. 2 we describe the numerical method, in Sect. 3 we present a verification of the method against an analytical solution and we also study a fractional RC electric circuit. The paper ends with some conclusions.

\section{Numerical Method}

Before presenting the numerical method, we will first present some preliminary results on the existence and uniqueness (see [2]) of solutions for system (1)-(2).

Lemma 1. Assume that the solution y of (1)-(2) exists and is unique on $[0, T]$, for a certain $T>0$. If $\alpha=\frac{p}{q}$, where $p \geq 1$ and $q \geq 2$ are two relatively prime integers and if each right-hand side function $F_{i}$ can be written in the form $F_{i}(t, \mathbf{y}(t))=\bar{F}_{i}\left(t^{1 / q}, \mathbf{y}(t)\right), i=1, \ldots, n-1$, where each $\bar{F}_{i}$ is analytic in a neighborhood of $\left(0, y_{i}(0)\right)$, then the components of the unique solution of 
the problem (1)-(2), $\mathbf{y}(t)=\left(y_{1}(t), \ldots, y_{n-1}(t)\right)$, can be represented in terms of powers of $t^{1 / q}$ :

$$
y_{i}(t)=\sum_{k=0}^{\infty} a_{k}^{i} t^{k / q}, t \in[0, r)
$$

where $r<T$ and $a_{k}^{i}$ are constants.

From the above Lemma it follows that for a fixed $m \in \mathbb{N}$ each component of the solution of (1)-(2) can be written as a sum $y_{i}(t)=y_{i}^{1}(t)+y_{i}^{2}(t), i=1, \ldots, n$, where, $y_{i}^{1} \in C^{m}([0, T]), i=1, \ldots, n$, and $y_{i}^{2}$ is the non-smooth part of $y_{i}$.

Note that if the order of the fractional derivative $\alpha$ is not of the form $\alpha=\frac{p}{q}$, with $p \geq 1$ and $q \geq 2$ two relatively prime integers, we can always replace $\alpha$ with the nearest rational number of this form, because rational numbers are dense in the reals, and, it has been proved in [6], that the solution of (1)-(2) depends continuously on the order of the derivative.

In order to approximate the solution of (1)-(2) we consider a nonuniform mesh on $[0, T]$, as in [7]. Given $N \in \mathbb{N}$, let $i_{0}$ be an integer such that $\left(\frac{N}{i_{0}}\right)^{\frac{m}{\alpha}} \leq N$ and $\left(\frac{N}{i_{0}-1}\right)^{\frac{m}{\alpha}}>N$ and let $N^{\prime}=N-i_{0}+1$. The partition on $[0, T]$ is defined through the meshpoints:

$$
t_{0}=0, t_{i}=\left(\frac{i_{0}+i-1}{N}\right)^{\frac{m}{\alpha}} T, \quad i=1,2, \ldots, N^{\prime}-1,
$$

and the $N^{\prime}$ subintervals:

$$
\sigma_{0}=\left[0, t_{1}\right], \quad \sigma_{i}=\left(t_{i}, t_{i+1}\right], i=1,2, \ldots, N^{\prime}-1
$$

with lengths $\tau_{i}=t_{i+1}-t_{i}, i=0,1, \ldots, N^{\prime}-1$. Define also $\tau=\max \tau_{i}$ with $i=0,1, \ldots, N^{\prime}-1$.

Consider the space

$$
\mathcal{V}_{m}^{\alpha}=\operatorname{span}\left\{t^{i+j \alpha}, i, j \in \mathbb{N}_{0}, i+j \alpha<m\right\}=\operatorname{span}\left\{t^{\nu_{k}}, k=1, \ldots, \ell\right\}, \quad \ell=\# \mathcal{V}_{m}^{\alpha}
$$

Taking Lemma 1 into account, if near the origin we approximate the solution of (1)-(2) with a function spanned by elements of space $\mathcal{V}_{m}^{\alpha}$, then it will reflect the potential non-smooth properties of the solution near the singularity. Therefore, we define the space

$$
S_{\tau}^{m}([0, T])=\left\{u \in C([0, T]):\left.\left.u\right|_{\sigma_{0}} \in \mathcal{V}_{m}^{\alpha}\right|_{\sigma_{0}},\left.\left.u\right|_{\sigma_{l}} \in \mathcal{P}_{m-1}\right|_{\sigma_{l}}, l=1, \ldots, N^{\prime}-1\right\},
$$

where $\mathcal{P}_{m-1}$ is the space of polynomials of degree less than or equal to $m-1$ and $\sigma_{i}, i=0,1, \ldots, N^{\prime}-1$, are defined by $(5)$.

On the first interval of the partition, $\sigma_{0}$, we define $\ell$ collocation points $t_{0 j}=$ $c_{j} \tau_{0}, j=1, \ldots, \ell, c_{j} \in[0,1]$, and on the remaining intervals $\sigma_{l}, l=1, \ldots, N^{\prime}-1$, 
we consider $m$ collocation points $t_{l j}=t_{l}+c_{j} \tau_{l}, \quad j=1, \ldots, m, c_{j} \in[0,1]$. Noting that each equation of system (1) can be written as [2],

$$
y_{i}(t)=y_{0 i}+\frac{1}{\Gamma(\alpha)} \int_{0}^{t}(t-s)^{\alpha-1}\left(\sum_{k=1}^{n} a_{i k} y_{k}(s)+f_{i}(s)\right) d s, i=1,2, \ldots, n
$$

we will then seek for a function $\mathbf{v}=\left[\begin{array}{lll}v_{1} & v_{2} \ldots v_{n}\end{array}\right]^{T}$ such that $v_{i} \in S_{\tau}^{m}([0, T])$, $i=1,2, \ldots, n$, that satisfies

$$
v_{i}\left(t_{0 j}\right)=y_{0 i}+\frac{1}{\Gamma(\alpha)} \int_{0}^{t_{0 j}}\left(t_{0 j}-s\right)^{\alpha-1}\left(\sum_{k=1}^{n} a_{i k} v_{k}(s)+f_{i}(s)\right) d s,
$$

where $j=1, \ldots, \ell$ if $p=0$ and $j=1, \ldots, m$ for $p=1, \ldots, N^{\prime}-1$. In order to obtain approximations for each $v_{i}\left(t_{0 j}\right), i=1, \ldots, n, j=1, \ldots, \ell$, we define the Lagrange functions, $\left.\left.\mathcal{L}_{0 j}\right|_{\sigma_{0}} \in \mathcal{V}_{m}^{\alpha}\right|_{\sigma_{0}}, j=1, \ldots, \ell$, such that $\mathcal{L}_{0 j}\left(t_{0 k}\right)=\delta_{j k}, k=$ $1, \ldots, \ell$. Then, we can write

$$
\mathcal{L}_{0 j}(t)=\sum_{i=1}^{\ell} \beta_{j i} t^{\nu_{i}}
$$

where, for each $j=1, \ldots, \ell$, the coefficients $\beta_{j i}$ may be obtained by solving the linear system $\mathcal{L}_{0 j}\left(t_{0 k}\right)=\delta_{j k}, k=1, \ldots, \ell$.

Hence, for $t \in \sigma_{0}$, we use the following representation for $\left.v_{i} \in \mathcal{V}_{m}^{\alpha}\right|_{\sigma_{0}}, i=$ $1, \ldots, n$ :

$$
y_{i}(t) \approx v_{i}(t)=\sum_{k=1}^{\ell} v_{i}\left(t_{0 k}\right) \mathcal{L}_{0 k}(t) .
$$

The values $v_{i}\left(t_{0 k}\right), k=1, \ldots, \ell$ are obtained by imposing that the functions $v_{i}(t)$ satisfy the integral Eq. (7), for $p=0$, at $t=t_{0 k}, k=1, \ldots, \ell$ :

$$
\begin{aligned}
v_{i}\left(t_{0 j}\right)= & y_{0 i}+\frac{1}{\Gamma(\alpha)} \int_{0}^{t_{0 j}}\left(t_{0 j}-s\right)^{\alpha-1}\left(\sum_{k=1}^{n} a_{i k} \sum_{p=1}^{\ell} v_{k}\left(t_{0 p}\right) \mathcal{L}_{0 p}(s)+f_{i}(s)\right) d s \\
& j=1, \ldots, \ell \\
y_{i}\left(t_{l k}\right)= & y_{0 i}+\frac{1}{\Gamma(\alpha)} \int_{0}^{t_{1}}\left(t_{l k}-s\right)^{\alpha-1}\left(\sum_{w=1}^{n} a_{i w} \sum_{p=1}^{\ell} y_{w}\left(t_{0 p}\right) \mathcal{L}_{0 p}(s)+f_{i}(s)\right) d s \\
+ & \frac{1}{\Gamma(\alpha)} \sum_{j=1}^{l-1} \int_{t_{j}}^{t_{j+1}}\left(t_{l k}-s\right)^{\alpha-1}\left(\sum_{p=1}^{n} a_{i p} y_{p}(s)+f_{i}(s)\right) d s \\
& +\frac{1}{\Gamma(\alpha)} \int_{t_{l}}^{t_{l k}}\left(t_{l k}-s\right)^{\alpha-1}\left(\sum_{p=1}^{n} a_{i p} y_{p}(s)+f_{i}(s)\right) d s \\
& i=1, \ldots, n, k=1, \ldots, m .
\end{aligned}
$$


On each subinterval $\sigma_{j}, j=1, \ldots, N^{\prime}-1$, each $y_{i}$ will be approximated by $v_{i} \in \mathcal{P}_{m-1}$, that is given by:

$$
y_{i}(t) \approx v_{i}(t)=\sum_{\gamma=1}^{m} L_{j \gamma}(t) v_{i}\left(t_{j \gamma}\right), \quad t \in \sigma_{j},
$$

where $L_{j \gamma}, j=1, \ldots, N^{\prime}-1, \gamma=1, \ldots, m$, are the Lagrange polynomials associated with the collocations points $t_{j \gamma}=t_{j}+\tau_{j} c_{\gamma}$.

Substituting in (7), for $p=1,2, \ldots, N^{\prime}-1$, we obtain the following system of equations, from which the values $v_{i}\left(t_{l k}\right), i=1, \ldots, n$, can be obtained:

$$
\begin{aligned}
v_{i}\left(t_{l k}\right) & =y_{0 i}+\frac{1}{\Gamma(\alpha)} \int_{0}^{t_{1}}\left(t_{l k}-s\right)^{\alpha-1}\left(\sum_{w=1}^{n} a_{i w} \sum_{p=1}^{\ell} v_{w}\left(t_{0 p}\right) \mathcal{L}_{0 p}(s)\right) d s \\
& +\frac{1}{\Gamma(\alpha)} \sum_{j=1}^{l-1} \int_{t_{j}}^{t_{j+1}}\left(t_{l k}-s\right)^{\alpha-1}\left(\sum_{p=1}^{n} \sum_{\gamma=1}^{m} a_{i p} L_{j \gamma}(s) v_{p}\left(t_{j \gamma}\right)\right) d s \\
& +\frac{1}{\Gamma(\alpha)} \int_{t_{l}}^{t_{l k}}\left(t_{l k}-s\right)^{\alpha-1}\left(\sum_{p=1}^{n} \sum_{\gamma=1}^{m} a_{i p} L_{l \gamma}^{(k)}(s) v_{p}\left(t_{l}+\tau c_{k} c_{\gamma}\right)\right) d s \\
& +\frac{1}{\Gamma(\alpha)} \int_{0}^{t_{l k}}\left(t_{l k}-s\right)^{\alpha-1} f_{i}(s) d s, \\
& l=1, \ldots, N^{\prime}-1, k=1, \ldots, m,
\end{aligned}
$$

where $L_{l \gamma}^{(k)}, l=1, \ldots, N^{\prime}-1, \gamma=1, \ldots, m$, are the Lagrange polynomials associated with the points $t_{l}+\tau_{l} c_{\gamma} c_{k}$.

After solving (8) and (9), the approximate solution of system (1)-(2), $\mathbf{v}=$ $\left[v_{i}\right]_{i=1}^{n}$ is given by:

$$
v_{i}(t)=\left\{\begin{array}{ll}
\sum_{k=1}^{\ell} v_{i}\left(t_{0 k}\right) \mathcal{L}_{0 k}(t), \quad t \in \sigma_{0} \\
\sum_{k=1}^{m} v_{i}\left(t_{j k}\right) L_{j k}(t), \quad t \in \sigma_{j}, j=1, \ldots, N^{\prime}-1
\end{array} .\right.
$$

Note that it was proved in the another work by the research group [5] that the convergence order of the method is $m$.

\section{$3 \quad$ Numerical Results}

In order to validate and illustrate the feasibility of the method, one example for which the analytical solution is known, and a case study involving fractional RC electric circuits are now presented. The numerical error is measured by determining the maximum error at the mesh points $t_{j}$ :

$$
\varepsilon_{\tau}=\max _{i=0, \ldots, N^{\prime}-1, j=1, \ldots, l_{i}}\left|y\left(t_{i j}\right)-v\left(t_{i j}\right)\right|,
$$

where $l_{0}=\ell$ and $l_{i}=m$ for $i=1, \ldots, N^{\prime}-1$, and, $v$ is the numerical solution. 


\subsection{Validation}

For validation purposes we have considered the following system:

$$
\left\{\begin{array}{l}
D^{\alpha} y_{1}(t)=y_{2}(t) \\
D^{\alpha} y_{2}(t)=-y_{1}(t)-y_{2}(t)+t^{\alpha+1}+\frac{\pi \csc (\pi \alpha) t^{1-\alpha}}{\Gamma(-\alpha-1) \Gamma(2-\alpha)}+\frac{\pi t \csc (\pi \alpha)}{\Gamma(-\alpha-1)},
\end{array}\right.
$$

together with the conditions $y_{1}(0)=0, y_{2}(0)=0$. The analytical solution is given by $y_{1}(t)=t^{1+\alpha}$ and $y_{2}(t)=\pi \alpha(\alpha+1) t \csc (\pi \alpha) / \Gamma(1-\alpha)$ with $\alpha \in[0,1]$.

For the numerical solution we consider a high order approximation with $m=4$. We consider Eq. (12) with several values of alpha: $\alpha=1 / 4,1 / 2,2 / 3$.

The numerical results listed in Table 1 suggest an experimental order of convergence of 4, as expected. Note that with standard finite difference schemes it would be impossible to attain such convergence rates. It should also be highlighted that fact that the new method is up to 338 times faster than the method in [4].

Table 1. Error and convergence order $(p)$ obtained for the numerical solution of Eq. (12). SU - simulation time of the non-polynomial method [4] (the solution is a linear combination of non-polynomial functions in the all domain) divided by the simulation time of the hybrid method.

\begin{tabular}{r|r|l|l|r|l|l|l|l|l|l|l}
\hline \multicolumn{2}{l|}{$\alpha=1 / 4$} & \multicolumn{4}{l}{$\alpha=1 / 2$} & \multicolumn{2}{l}{$\alpha=2 / 3$} \\
\hline \multicolumn{1}{c|}{$N^{\prime}$} & $N^{\prime}$ & $\varepsilon_{\tau}$ & $p$ & \multicolumn{1}{l|}{$N^{\prime}$} & $\varepsilon_{\tau}$ & $p$ & $\mathrm{SU}$ & $N^{\prime}$ & $\varepsilon_{\tau}$ & $p$ & $\mathrm{SU}$ \\
\hline 64 & 15 & $2.11 \cdot 10^{-6}$ & - & 26 & $1.94 \cdot 10^{-7}$ & - & 56 & 32 & $5.53 \cdot 10^{-8}$ & - & 142 \\
\hline 128 & 34 & $1.45 \cdot 10^{-7}$ & 3.86 & 59 & $1.27 \cdot 10^{-8}$ & 3.93 & 50 & 71 & $3.58 \cdot 10^{-9}$ & 3.95 & 162 \\
\hline 256 & 75 & $9.43 \cdot 10^{-9}$ & 3.94 & 128 & $8.13 \cdot 10^{-10}$ & 3.97 & 52 & 155 & $2.28 \cdot 10^{-10}$ & 3.97 & 170 \\
\hline 512 & 166 & $6.00 \cdot 10^{-10}$ & 3.97 & 278 & $5.14 \cdot 10^{-11}$ & 3.98 & 59 & 331 & $1.44 \cdot 10^{-11}$ & 3.99 & 338 \\
\hline
\end{tabular}

\subsection{Case Study: Fractional RC Electrical Circuits}

By following the work of Gómez-Aguilar et al. [3] the fractional differential equation governing a fractional RC electrical circuit (see Fig. 1(a)) is given by (for more details see [3]),

$$
\frac{d^{\alpha} q(t)}{d t^{\alpha}}+\alpha^{1-\alpha}(R C)^{-\alpha} q(t)=C \alpha^{1-\alpha}(R C)^{-\alpha} E(t), \alpha \in[0,1] .
$$

We have solved the governing equation using the hybrid method (the parameters are shown in Fig. 1).

Note that we have considered the relationship given by Eq. (12) of [3], stating that $\sigma_{R}=\alpha R C$.

We compared our results with the results obtained with the numerical Laplace transform technique, where an excellent agreement is observed. Note that we use $\alpha=0.96,0.98$ which are close to 1 (the classical case), but, huge differences between the two values were observed in [3]. 


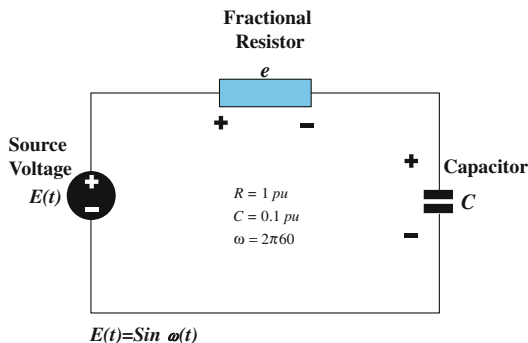

(a)

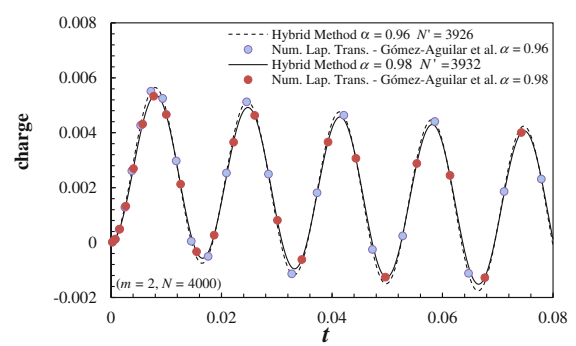

(b)

Fig. 1. (a) Schematic of the RC circuit. (b) Charge vs. time. Comparison with the results obtained by Gómez-Aguilar et al. [3] (the parameters used in the numerical solution of Eq. (13) are given in the figure). The simulations were performed with Mathematica 11.0 from Wolfram Research, Inc.

\section{Conclusions}

A hybrid numerical method was developed for the solution of systems of fractional differential equations. The method can deal with both regular and singular solutions extending in this way the results in [4] where only one equation was considered and where the non-polynomial approximation was used along all the time interval. The numerical results suggest that the computational effort of the new method is significantly lower and that it provides an optimal convergence order $m$ that is independent of $\alpha$. The hybrid method is also used to study fractional $\mathrm{RC}$ electrical circuits, as an alternative to the Numerical Laplace Transform.

Acknowledgments. The first, third and fourth authors would like to thank the funding by FCT-Portuguese Foundation for Science and Technology through scholarship and projects: SFRH/BPD/100353/2014 and UID/Multi/04621/2013, UID/MAT/00297/2013 (Centro de Matemática e Aplicações), respectively.

\section{References}

1. Podlubny, I.: Fractional Differential Equations: An Introduction to Fractional Derivatives, Fractional Differential Equations, to Methods of Their Solution and Some of Their Applications. Mathematics in Science and Engineering (1999)

2. Diethelm, K.: The Analysis of Fractional Differential Equations: An ApplicationOriented Exposition Using Differential Operators of Caputo Type. Springer, Heidelberg (2010)

3. Gómez-Aguilar, J.F., Rosales-García, J., Razo-Hernández, J.R., Guía-Calderón, M.: Fractional RC and LC electrical circuits. Ing. Inv. y Tec. 15, 311-319 (2014)

4. Ford, N.J., Morgado, M.L., Rebelo, M.: Non-polynomial collocation approximation of solutions to fractional differential equations. Fract. Calc. Appl. Anal. 16, 874-891 (2013) 
5. Ferrás, L.L., Ford, N.J., Morgado, M.L., Rebelo, M.: High-orders Methods for Systems of Fractional Ordinary Differential Equations and their Application to TimeFractional Diffusion Equations (2018, to be submitted)

6. Diethelm, K., Ford, N.J.: Analysis of fractional differential equations. J. Math. Anal. Appl. 265, 229-248 (2002)

7. Cao, Y., Herdman, T., Xu, Y.: A hybrid collocation method for volterra integral equations with weakly singular kernels. SIAM J. Numer. Anal. 41, 364-381 (2003) 